\title{
Diagnostic Utility of Diffusion Tensor Imaging in Differentiating Glioblastomas from Brain Metastases
}

\author{
S. Wang, S.J. Kim, H. Poptani, J.H. Woo, S. Mohan, R. Jin, M.R. Voluck, D.M. O'Rourke, R.L. Wolf, E.R. Melhem, and S. Kim
}

\begin{abstract}
BACKGROUND AND PURPOSE: Differentiation of glioblastomas and solitary brain metastases is an important clinical problem because the treatment strategy can differ significantly. The purpose of this study was to investigate the potential added value of DTI metrics in differentiating glioblastomas from brain metastases.
\end{abstract}

MATERIALS AND METHODS: One hundred twenty-eight patients with glioblastomas and 93 with brain metastases were retrospectively identified. Fractional anisotropy and mean diffusivity values were measured from the enhancing and peritumoral regions of the tumor. Two experienced neuroradiologists independently rated all cases by using conventional MR imaging and DTI. The diagnostic performances of the 2 raters and a DTI-based model were assessed individually and combined.

RESULTS: The fractional anisotropy values from the enhancing region of glioblastomas were significantly higher than those of brain metastases $(P<.01)$. There was no difference in mean diffusivity between the 2 tumor types. A classification model based on fractional anisotropy and mean diffusivity from the enhancing regions differentiated glioblastomas from brain metastases with an area under the receiver operating characteristic curve of 0.86 , close to those obtained by 2 neuroradiologists using routine clinical images and DTI parameter maps (area under the curve $=0.90$ and 0.85 ). The areas under the curve of the 2 radiologists were further improved to 0.96 and 0.93 by the addition of the DTI classification model.

CONCLUSIONS: Classification models based on fractional anisotropy and mean diffusivity from the enhancing regions of the tumor can improve diagnostic performance in differentiating glioblastomas from brain metastases.

ABBREVIATIONS: $A U C=$ area under the curve; $E R=$ enhancing region; $F A=$ fractional anisotropy; $I P R=$ immediate peritumoral region; $L R M=$ logistic regression model; $M D=$ mean diffusivity

D ifferentiation of glioblastomas and solitary brain metastases is an important clinical problem because the treatment strategy can significantly differ depending on the tumor type. ${ }^{1,2}$ In some cases, clinical history and/or multiplicity of enhancing brain lesions makes the diagnosis of brain metastases relatively straight-

Received July 8, 2013; accepted after revision September 13.

From the Departments of Radiology (S.W., H.P., J.H.W., S.M., M.R.V., R.L.W.) and Neurosurgery (D.M.O.), Hospital of the University of Pennsylvania, Philadelphia, Pennsylvania; Department of Radiology (S.J.K.), University of Ulsan, Asan Medical Center, Seoul, Republic of Korea; Medical Data Research Center (R.J.), Providence Health and Services, Portland, Oregon; Department of Diagnostic Radiology and Nuclear Medicine (E.R.M.), University of Maryland Medical Center, Baltimore, Maryland; and Department of Radiology (S.K.), New York University School of Medicine, New York, New York.

Paper previously presented in part at: Annual Meetings of the International Society for Magnetic Resonance in Medicine, May 5-11, 2012; Melbourne, Victoria, Australia; and April 20-26, 2013; Salt Lake City, Utah.

Please address correspondence to Sumei Wang, MD, Department of Radiology, Division of Neuroradiology, Hospital of the University of Pennsylvania, 219 Dulles Building, 3400 Spruce St, Philadelphia, PA 19104; e-mail: Sumei.Wang@uphs.upenn.edu

http://dx.doi.org/10.3174/ajnr.A3871 forward. However, a solitary brain metastasis on MR imaging can have a nonspecific appearance. Similarly confounding the issue is the fact that glioblastomas can also occasionally present as multiple enhancing lesions. Moreover, although a glioblastoma typically presents as a solitary mass, a solitary brain metastasis may be the first manifestation of disease in approximately $30 \%$ of patients with systemic cancer. ${ }^{3}$ Hence, accurate distinction between glioblastomas and brain metastases can be challenging, often necessitating an invasive surgical biopsy for a definitive diagnosis.

DTI has been used to differentiate glioblastomas from brain metastases, but with conflicting results. Some reports have suggested that mean diffusivity $(\mathrm{MD})^{4-6}$ is helpful for the differentiation, while others indicated the limited use of MD in the differentiation of neoplasms. ${ }^{7-9}$ Wang et $\mathrm{al}^{10}$ and Reiche et $\mathrm{al}^{11}$ reported lower fractional anisotropy (FA) from the enhancing regions (ERs) of glioblastomas compared with brain metastases. In contrast, another study reported that glioblastomas have higher FA in the enhancing regions than metastases. ${ }^{12}$ The potential reasons for these conflicting results may include differ- 
ences in analysis methods, particularly in defining the region of interest, as well as the relatively small size of cohorts used in individual studies $(n=24-66) .^{4,5,7,10,13}$ Hence, a primary objective of this study was to investigate the DTI characteristics of glioblastomas and brain metastases in a substantially larger cohort of patients, in order to determine whether they may have diagnostic utility.

Restricted water diffusion in solid tumors has been reported by many groups. ${ }^{7,14-17}$ It is generally assumed that a solid tumor does not have any microstructural orientation by itself and its growth induces structural disorder in the tissue, which leads to decreased FA. ${ }^{18}$ However, although the association between FA and tumor cellularity has been studied, the relationship remains unclear because both positive ${ }^{12,14,19}$ and negative $^{16}$ correlations have been reported. Our previous study $^{12}$ showing higher FA in glioblastomas than in brain metastases also found a negative linear trend between FA and MD in glioblastomas, which was not seen in metastases, perhaps indicating a tumor-dependent relationship between these 2 parameters. While promising results with DTI have been published in the diagnosis of brain tumors, to date, its clinical value in improving the diagnostic accuracy of radiologists has not been evaluated.

Hence, the objectives of the present study were to investigate the potential of DTI in differentiation of glioblastomas from solitary brain metastases in a relatively large cohort of 221 patients and to compare its diagnostic accuracy in comparison with that of 2 experienced neuroradiologists.

\section{MATERIALS AND METHODS Patients}

Patients with solitary enhancing lesions $(n=221)$, based on contrast-enhanced T1-weighted images, were retrospectively recruited from our institutional data base between June 2006 and February 2012. Patients with nonenhancing or multiple enhancing tumors or a clinical history of any prior therapy to the brain were not included. The study was approved by the institutional review board and was compliant with the Health Insurance Portability and Accountability Act.

Histopathologic diagnosis of the tumor was available for all patients through surgical resection or biopsy. The final diagnoses included 128 glioblastomas ( 78 men, 50 women; age, $60.5 \pm 12.3$ years; range, 24-90 years) and 93 brain metastases (46 men, 47 women; age, $58.8 \pm 11.6$ years; range, $44-88$ years). Of the 93 metastases, the primary sites for cancer included lung $(n=56)$, breast $(n=16)$, skin $(n=8)$, colon $(n=3)$, renal $(n=2)$, neck $(n=1)$, arm $(n=1)$, parotid $(n=1)$, esophagus $(n=2)$, thyroid $(n=1)$, peritoneum $(n=1)$, and endometrium $(n=1)$.

\section{Data Acquisition}

MR imaging studies were performed on a Tim Trio 3T wholebody scanner (Siemens, Erlangen, Germany) by using a 12-channel phased-array head coil. Routine MR imaging pulse sequences included axial T1-weighted 3D MPRAGE (TR/TE/TI = 1760/3.1/ $950 \mathrm{~ms}$, matrix size $=192 \times 256$, section thickness $=1 \mathrm{~mm})$ and axial FLAIR $(\mathrm{TR} / \mathrm{TE} / \mathrm{TI}=9420 / 141 / 2500 \mathrm{~ms}$, section thickness $=$ $3 \mathrm{~mm}$ ). DTI data were acquired by using a single-shot spin-echo
EPI sequence with parallel imaging by using generalized autocalibrating partially parallel acquisition and an acceleration factor of 2. The DTI data from 55 patients ( 40 glioblastomas, 15 metastases) were acquired by using 12 diffusion-weighting directions $(\mathrm{TR} / \mathrm{TE}=4900 / 83 \mathrm{~ms}, \mathrm{NEX}=6)$; and in the remaining 166 patients, DTI data were acquired with 30 directions (TR/TE $=$ $5000 / 86 \mathrm{~ms}, \mathrm{NEX}=3$ ). Other sequence parameters were as follows: $\mathrm{FOV}=22 \times 22 \mathrm{~cm}^{2}, b=0,1000 \mathrm{~s} / \mathrm{mm}^{2}$, section thickness $=3 \mathrm{~mm}$, total scanning time $=8$ minutes. All our 12 - and 30 -direction DTI data were acquired by using the same imaging parameters with the same scanning times at the same scanner. On the basis of previous studies, ${ }^{20-22}$ we assume that there is no significant difference in mean or median MD and FA values from the 12- and 30-direction DTI datasets, particularly when FA is mostly $<0.3$ as in the solid tumors included in this study ${ }^{21}$ and we combined the data from all the patients in this study.

\section{Image Processing}

The diffusion-weighted images were coregistered to the non-diffusion-weighted $(b=0)$ images by using the methods described in another work. ${ }^{12}$ The corrected raw images were combined to estimate rotationally invariant DTI parameter maps, including FA and MD, by using in-house software. ${ }^{12,23,24}$

The DTI parameter maps and FLAIR images were then coregistered to contrast-enhanced T1-weighted images. A semiautomatic segmentation approach was used to subdivide each lesion into 3 regions: enhancing region, immediate peritumoral region (IPR), and distant peritumoral region by using contrast-enhanced T1 and FLAIR images. ${ }^{12}$ ER was the region with enhancement higher than the mean +3 SDs of the signal intensity from the WM. IPR was chosen as a 4-mm-wide band around the enhancing region. The remaining region of FLAIR abnormality, outside of the IPR, was the distant peritumoral region. ${ }^{12,23,25}$ The median FA and MD values were measured from the 3 regions. Data analysis tools, including image coregistration and segmentation, were implemented by using IDL (Exelis Visual Information Solutions, Boulder, Colorado).

\section{Data Analysis}

A Mann-Whitney $U$ test was used for the difference in the median MD and FA values between glioblastomas and brain metastases from the 3 regions. A $P$ value $<.05$ was considered significant. Linear regression analysis was used to investigate the association between 2 continuous measures, FA and MD. A multivariate logistic regression analysis was used to determine the best classification model of DTI parameters, namely the logistic regression model (LRM). Bootstrapping validation was applied to estimate the accuracy of the LRM. Specifically, $70 \%$ of the full dataset was randomly selected with replacement and was used as an independent validation set; this process was repeated 500 times. ${ }^{26}$ Means and SDs of the area under the receiver operating characteristic curves (AUCs) were computed. The cutoff lines for separating glioblastomas from metastases were determined by maximizing the sum of specificity and sensitivity (ie, Youden index).

Two neuroradiologists with 10 and 9 years of experience independently reviewed the cases on the basis of contrast-enhanced 

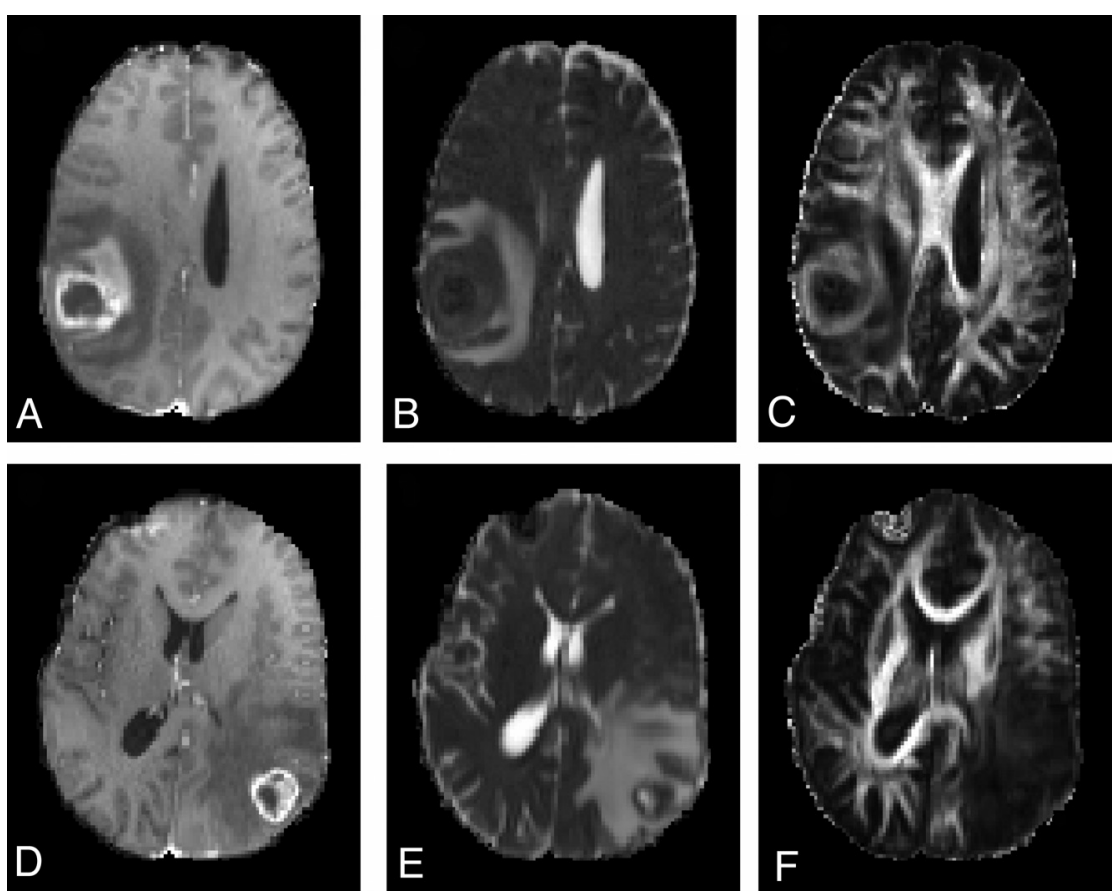

FIG 1. Comparison of imaging features between glioblastomas $(A-C)$ and brain metastases $(D-F)$. Both show ring enhancement and extensive edema on axial contrast-enhanced T1-weighted images $(A$ and $D)$ and restricted diffusion of the enhancing part on MD maps ( $B$ and $E$ ). However, for the FA map, the glioblastoma case demonstrates high FA values from the enhancing region. The high FA starts from the enhancing region and extends to the immediate peritumoral region, making an FA rim.

T1, FLAIR, DWI, FA, and MD maps. The radiologists were asked to rate their level of confidence for each case, whether the tumor was metastasis or glioblastoma. A rating was classified into 5 scales based on the following 5 confidence levels: level 1,90\% metastasis; level 2, 70\% metastasis; level 3, 50\%; level 4, 70\% glioblastoma; and level 5, 90\% glioblastoma. The diagnostic performances of the 2 raters and LRM were evaluated by using the receiver operating characteristic analysis curves. The AUCs were compared by using the method of DeLong et al. ${ }^{27}$ The weighted $\kappa$ was used to assess the interobserver agreement between the 2 raters and LRM. All data analysis was conducted by using the Statistical Package for the Social Sciences for Windows, Version 15.0 (IBM, Armonk, New York). R software (Version 2.15.0; http:// www.r-project.org) was used for the bootstrapping validation.

\section{RESULTS}

\section{Comparison of Imaging Parameters}

Representative MR images of patients with glioblastoma and metastasis are shown in Fig 1. The MD maps in both cases showed similar levels of restricted diffusion in the enhancing parts. In contrast, the FA maps showed that the glioblastoma had higher FA than the brain metastasis. The high FA rim extended to the immediate peritumoral regions in glioblastomas. Similar observation was made with most cases as summarized by the boxplots shown in Fig $2 A,-B$. The median FA value of glioblastomas from the ER and IPR was significantly higher than that of brain metastases $(P<.01)$. There was no significant difference in the median values of MD from all the regions. None of the parameters showed significant differences from the distant peritumoral regions.

\section{Diffusion Tensor Logistic Regression Model}

FA from the ER and IPR and MD from the ER were evaluated for their diagnostic performance by using receiver operating characteristic analysis (Table). The single best predictor for the discrimination is FA from the ER with a sensitivity of 0.80 , a specificity of 0.76 , and an AUC of 0.84 . The logistic regression analysis indicated that the best model included FA and MD from the ER as follows:

1) $f(M D, F A)$

$$
=\frac{1}{1+\exp [-(\beta 0+\beta 1 M D+\beta 2 F A)]},
$$

where $\beta 0=-7.79, \beta 1=2.14$, and $\beta 2=$ 43.30. This model was validated by using the bootstrapping procedure described above (repeated 500 times) with a mean AUC of 0.86 and SD of 0.03 .

The scatterplots of FA and MD from the ER in Fig $2 C$ demonstrate how LRM can have a higher AUC than FA alone, though $\mathrm{MD}$ itself is a poor predictor $($ AUC $=0.50)$. The cutoff line of LRM shown in Fig $2 C$ was slanted and positioned between the linear regression lines for glioblastomas and brain metastases, shown separately in Fig $2 D,-E$, respectively. The linear negative regression between FA and $\mathrm{MD}$ in the glioblastoma group was significant $\left(R^{2}=0.26, P<\right.$ $.05)$.

\section{Comparison between Diffusion Tensor and Raters}

The results of diagnostic reading by 2 expert neuroradiologists by using the 5-point rating scale and LRM output are presented as scatterplots in Fig $3 A,-B$. If one assumes that the LRM output is equivalent to the reader confidence level, confidence level 1 of the raters ( $90 \%$ metastasis) corresponds to $0-0.2$ of the LRM output; confidence level 2, to $0.2-0.4$ of the LRM output; and so forth. The weighted $\kappa$ values between the 2 raters, rater 1 and LRM, and rater 2 and LRM, were $0.63,0.40 .0 .43$, respectively, indicating moderate agreement. The receiver operating characteristic analysis curves in Fig $3 C$ demonstrate that the diagnostic performances of the 2 raters and LRM are similar; the AUC values were 0.90, 0.85 , and 0.86 , for rater 1 , rater 2 , and LRM, respectively. There was a significant difference between the AUCs of rater 1 and rater $2(P=.03)$.

Figure $3 A,-B$ indicates that approximately half of the cases had low confidence levels (levels $2-4$ for the raters and $0.2-0.8$ for the LRM): $43 \%$ for rater 1, 56\% for rater 2, and 50\% for the LRM. Fig $3 D$ shows the receiver operating characteristic analysis curves only for the cases with confidence levels between 2 and 4 by both raters $(n=72)$. The AUC of the LRM ( 0.87$)$ for these challenging cases was higher than those of rater $1(0.70)$ and rater $2(0.64)$, suggesting that the LRM can be helpful in improving the classification of the cases with low rater confidence levels. 

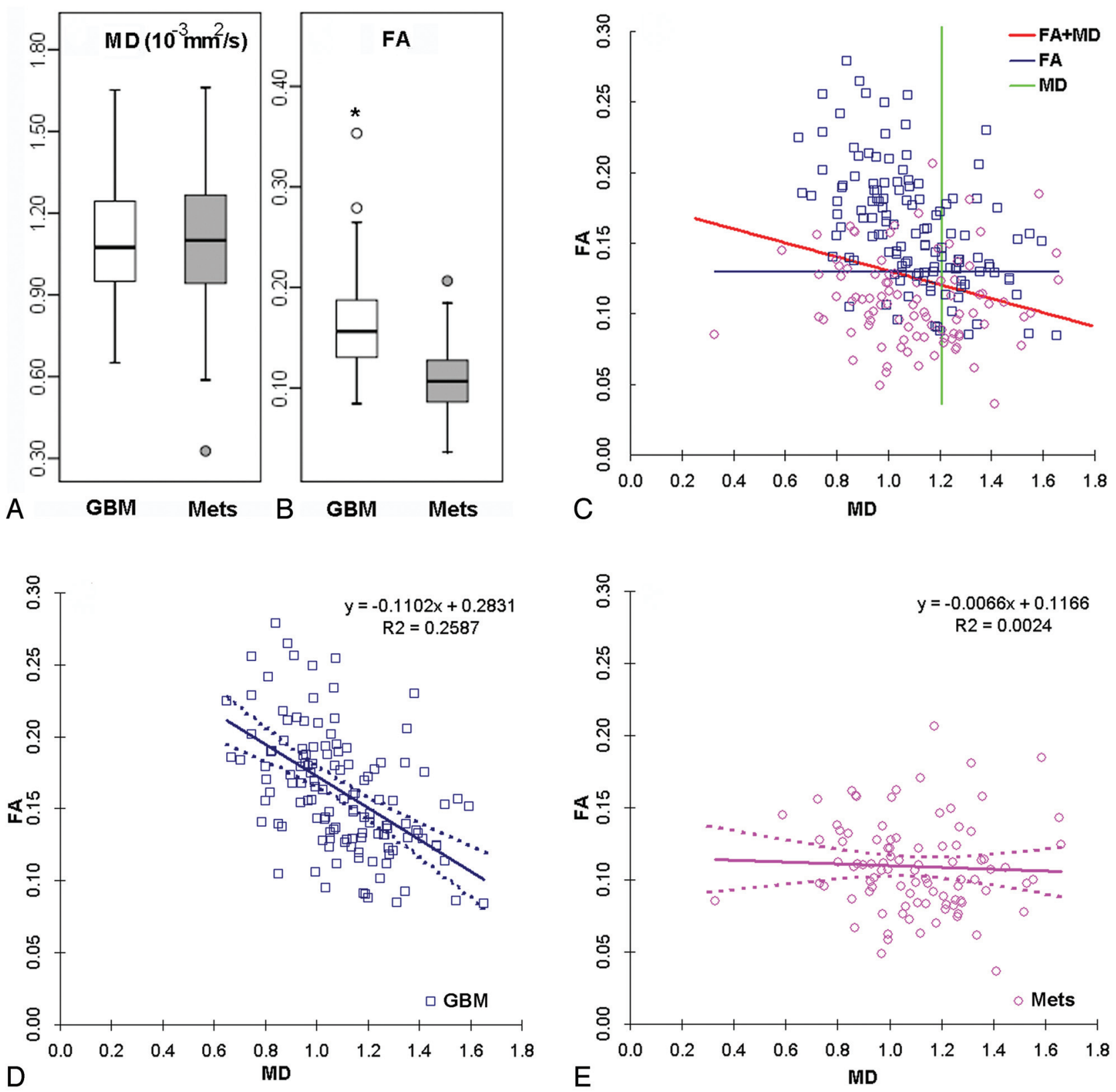

FIG 2. Boxplot of FA and MD from the enhancing region in glioblastomas (white) and brain metastases (gray) ( $A$ and $B$ ). The outliers are represented by circles. Asterisks indicate significant differences $(P<.01)$. A scatterplot of FA and MD from the enhancing region of glioblastomas (blue square) and brain metastases (purple circle) (C) is shown. The green line represents the cutoff line of MD; the blue line, the cutoff line of FA; and the red line, the cutoff line of the combined model of FA and MD, which can successfully separate the glioblastomas and brain metastases. FA and MD regression lines for glioblastomas (D), FA and MD regression lines for brain metastases $(E)$, and the dotted line indicate $95 \%$ confidence intervals. There is a negative correlation of FA and MD in glioblastomas $(R=0.51, P<.05)$.

Diagnostic performance of 2 raters and the logistic regression model of DTI parameters for all the cases

\begin{tabular}{|c|c|c|c|c|c|c|}
\hline & Sensitivity & Specificity & PPV & NPV & AUC & Cutoff Value \\
\hline$F A_{E R}$ & 0.80 & 0.76 & 0.80 & 0.73 & 0.84 & 0.13 \\
\hline$M D_{\mathrm{ER}}\left(10^{-3} \mathrm{~mm}^{2} / \mathrm{s}\right)$ & 0.73 & 0.34 & 0.60 & 0.47 & 0.51 & 1.21 \\
\hline$F A_{I P R}$ & 0.65 & 0.68 & 0.73 & 0.58 & 0.69 & 0.17 \\
\hline LRM & 0.84 & 0.77 & 0.83 & 0.78 & 0.86 & 0.50 \\
\hline Rater 1 & 0.70 & 0.93 & 0.75 & 0.94 & 0.90 & 4.5 \\
\hline LRM + rater 1 & 0.93 & 0.88 & 0.92 & 0.90 & 0.96 & 0.54 \\
\hline Rater 2 & 0.76 & 0.85 & 0.94 & 0.54 & 0.85 & 2.5 \\
\hline LRM + rater 2 & 0.85 & 0.85 & 0.88 & 0.81 & 0.93 & 0.54 \\
\hline
\end{tabular}

Note:-PPV indicates positive predictive value; NPV, negative predictive value. 

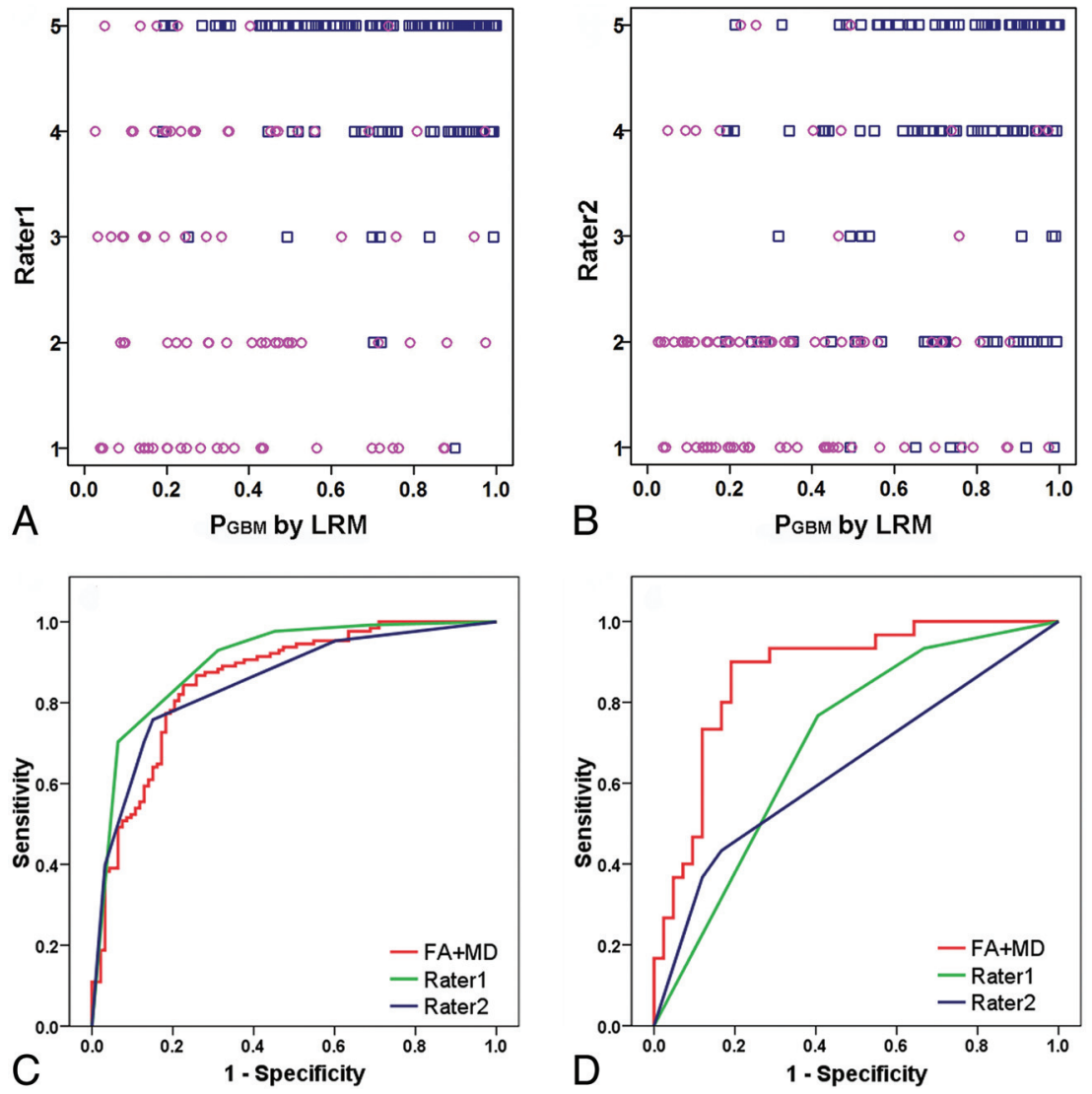

FIG 3. Scatterplot of 2 raters and the logistic regression model for glioblastomas (blue square) and brain metastases (purple circles) ( $A$ and $B$ ). Approximately half of cases were with low confidence levels (levels $2-4$ for the raters and $0.2-0.8$ for the $L R M) . P_{G B M}$ represents the probability, predicted by the model, for glioblastoma (GBM). Receiver operative characteristic curve analysis from 2 raters and the LRM for the whole cases $(C)$ and challenging cases for the raters with confidence level of 2-4 (D) are shown. The performance of the LRM is close to that of both raters. For challenging cases, the performance of the LRM remains at about the same level as those for all cases.

\section{DTI and Rater Combined Classifier}

Figure 4 illustrates the improvement in the diagnostic accuracy of each rater by combining with the LRM. A logistic regression model was generated by using LRM output and rater 1 :

\section{2) $f($ Rater $1, L R M)=\frac{1}{1+\exp [-(\beta 0+\beta 1 \text { Rater } 1+\beta 2 L R M)]}$}

where $\beta 0=-9.54, \beta 1=1.70$, and $\beta 2=6.25$. The scatterplot in Fig $4 A$ demonstrates that most of the glioblastomas are above the diagonal line and most of the brain metastases are below the diagonal line. LRM plus rater 1 had an AUC of 0.96 compared with 0.9 by rater 1 alone. We tested the model by using rater 2 . Figure $4 B$, - $D$ shows that the combined model with LRM plus rater 1 can also improve the results of rater 2. The AUC was 0.93 , compared with 0.85 by rater 2 alone. The combined model significantly improved the AUC of both rater $1(P<.001)$ and rater $2(P<.001)$. This model can be applied to all raters.

A summary of diagnostic performance of individual readers and combined schemes of DTI parameters, including sensitivity, specificity, positive predictive value, negative predictive value, and AUC, is shown in the Table.

\section{DISCUSSION}

In this study, the diagnostic performance of DTI based on FA and MD was compared with those of 2 experienced neuroradiologists. Our results demonstrated that the diagnostic accuracy of the classifier was as good as those of experienced neuroradiologists and that LRM could be used to further improve the diagnostic performance of the readers. Our previous studies in a smaller sample population $(n=63)$ have shown that FA and MD from the enhancing part are very useful for differentiating glioblastomas from brain metastases. ${ }^{12,23}$ Results from the present study with a substantially larger sample size ( $n=221)$ confirmed that FA and MD from the enhancing part can be used to generate a robust model for the classification between glioblastomas and brain metastases.

Our study demonstrated that FA from the enhancing regions of glioblastomas was significantly higher than that of brain metastases. Toh et $\mathrm{al}^{28,29}$ reported similar findings and interpreted them as the influence of gliosis surrounding the glioblastomas. We speculate that the high FA in glioblastomas may be due to the orientation of overproduced extracellular matrix. ${ }^{30,31}$ The extracellular space and matrix play an important role in tumor growth and infiltration. Glioblastoma cells produce large amounts of tumor-specific extracellular matrix components, which serve as a substrate for adhesion and subsequent migration of the cells through the enlarged extracellular space. ${ }^{30}$ These molecules accumulate and are oriented in extracellular matrix, ${ }^{32}$ resulting in high anisotropy. Our previous study in meningiomas demonstrated high FA and planar anisotropy coefficient in fibroblastic meningiomas compared with other subtypes. ${ }^{26}$ These findings can be explained by the presence of a large amount of collagen in fibroblastic subtypes. ${ }^{25,33}$ It has been reported that the structure and orientation of extracellular matrix affect the anisotropy, but not the mean diffusivity $^{34}$; these results are consistent with ours. On the other hand, in brain metastases, neoplasm cells grow into the brain parenchyma in an expansive, noninfiltrating pattern. In contrast to glioblastoma, degradation of the extracellular matrix begins the process of metastasis. ${ }^{35,36}$

We did not observe a significant difference in MD values from the enhancing region between glioblastomas and metastases, indicating a limited sensitivity and specificity of this parameter in tumor differentiation. However, MD was still an important parameter for LRM because FA in glioblastomas varied depending on $\mathrm{MD}$, but did not in metastases. If one assumes that MD reflects 

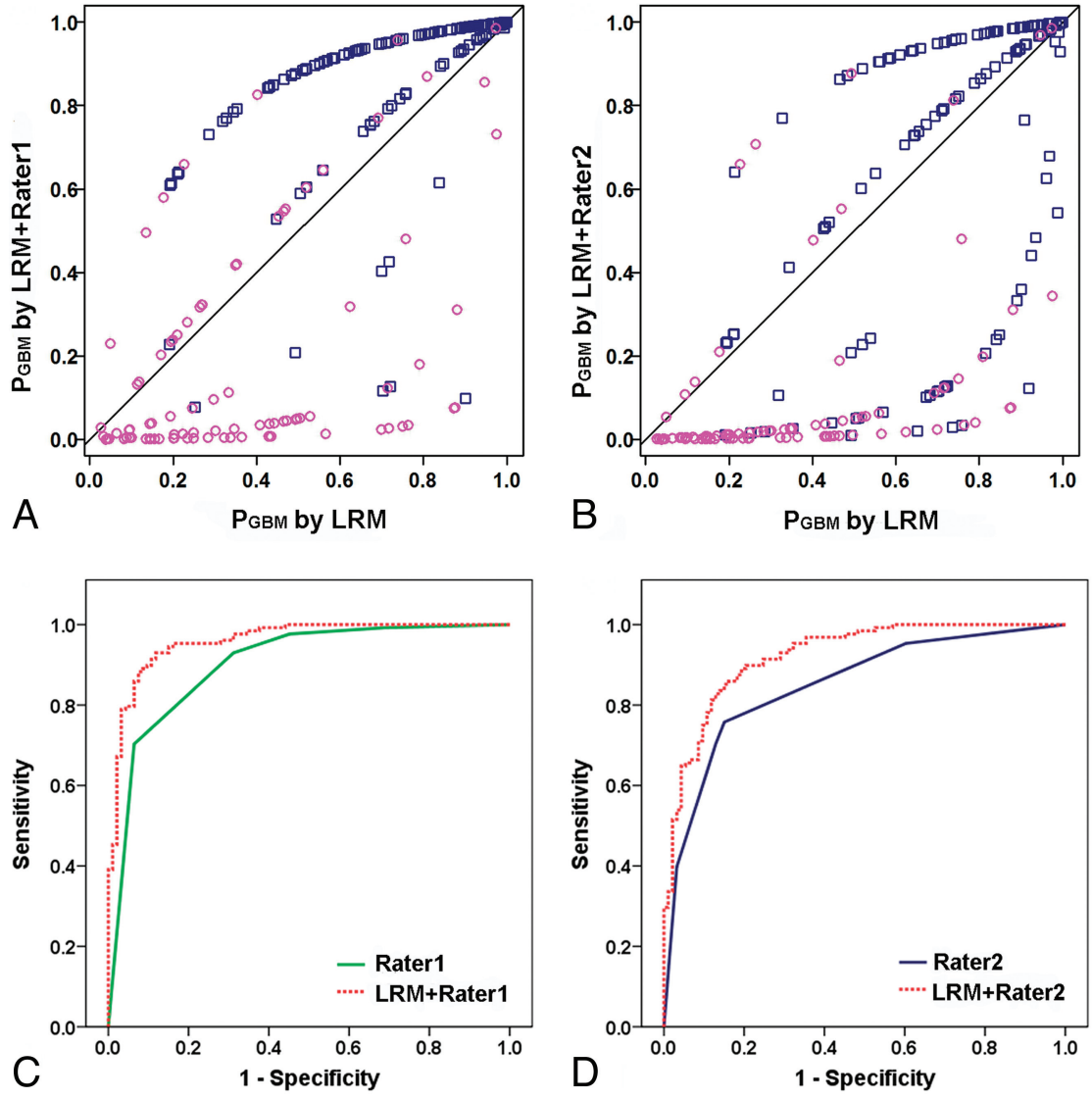

FIG 4. The diagnostic performance of rater 1 improves by combining the DTI logistic regression model ( $A$ and $C$ ). Most of the glioblastomas (blue squares) are above the diagonal line, and most of the brain metastases (purple circles) are below the diagonal line. $\mathrm{P}_{\mathrm{GBM}}$ represents the probability, predicted by the model, for glioblastoma (GBM). AUC improves from 0.90 to 0.960 for rater 1. The combined model with LRM plus rater 1 can also improve the results of rater 2 ( $B$ and $D$ ). AUC improves from 0.85 to 0.93 for rater 2 .

cellularity, our findings from in vivo data substantiate previous reports on the positive correlation between FA and tumor cellularity. ${ }^{12,14,19}$ Furthermore, our study shows, for the first time, that such association between FA and MD is found only in glioblastomas, but not in brain metastases. The underlying mechanism for such discrepancy between these 2 types of tumors is not fully understood, but it may help in improving the diagnostic accuracy as demonstrated in this study.

In this study, the AUC values are similar between the raters (0.90 and 0.85) and LRM (0.86). This result indicates that the 2 raters and LRM had differences in their confidence levels for individual cases, but overall the performance of LRM was close to that of both raters. Furthermore, with the use of the DTI classification model, the diagnostic performance of each radiologist improved noticeably, with high sensitivity, specificity, positive predictive values, and negative predictive values. The AUC increased from 0.90 to 0.96 for rater 1 and from 0.85 to 0.93 for rater 2 . The model is easy to use in clinical practice. Specifically, the readers first interpreted the case on the basis of the 5-scale confidence levels. Then they put the FA and MD values from the enhancing part into equation 1 and calculated the probability. Finally they used equation 2 to get the final interpretation. Alternatively, on the basis of the promising results of the current study, the LRM output can be provided to the radiologists as complementary information to consider when reading the case. A future study is warranted to test whether the availability of the LRM output can improve the diagnostic performance of radiologists with different levels of experience. We also evaluated challenging cases for the readers with lower confidence levels. The AUC remained at about the same level as that in all the cases. This finding was in accordance with several previous studies $^{5,12,17}$ and indicates that the model is very helpful for readers, even for experienced radiologists.

A potential limitation of our study is its retrospective design. To further validate our results, a prospective study with similar or larger cohort size is warranted. Water diffusivity is affected by many factors including cellularity, extracellular volume, viscosity, and membrane permeability. The mean diffusivity and directionality changes measured by DTI are the sum of all the microstructural changes. Hence, a thorough pathologic investigation would be necessary to elucidate the underlying tissue structure responsible for higher FA in glioblastomas and the association with MD. In this retrospective study, image-guided biopsy was not available. Future study with pathologic validation is required. Furthermore, we may further improve the DTI-based model by using histogram analysis of FA and MD values in voxels. Future study is warranted to fully use the vast voxelwise information collected through DTI for better diagnosis.

\section{CONCLUSIONS}

The classification model based on FA and MD from the enhancing region is a robust one for differentiating glioblastomas from brain metastases. The model performed as well as experienced neuroradiologists. The diagnostic performance of radiologists in differentiating glioblastomas from brain metastases can be improved by adding a DTI classification model.

Disclosures: Harish Poptani-UNRELATED: Consultancy: American College of Radiology Image Metrix, Grants/Grants Pending: National Institutes of Health.* Donald M. O'Rourke-UNRELATED: Expert Testimony: neurosurgery medicolegal expert review, Grants/Grants Pending: Celldex Therapeutics,* Philanthropy,* Patents (planned, pending or issued): US Patents, University of Pennsylvania.* Ronald L. Wolf-UNRELATED: Other: ACRIN “tie-breaker" reads for glioblastoma study, Comments: unrelated to publication. ${ }^{*}$ Money paid to the institution.

\section{REFERENCES}

1. Giese A, Westphal M. Treatment of malignant glioma: a problem beyond the margins of resection. J Cancer Res Clin Oncol 2001;127:217-25

2. Soffietti R, Ruda R, Mutani R. Management of brain metastases. J Neurol 2002;249:1357-69

AJNR Am J Neuroradiol 35:928-34 May 2014 www.ajnr.org 
3. Schiff D. Single brain metastasis. Curr Treat Options Neurol 2001;3:89-99

4. Lu S, Ahn D, Johnson G, et al. Peritumoral diffusion tensor imaging of high-grade gliomas and metastatic brain tumors. AJNR Am J Neuroradiol 2003;24:937-41

5. Lu S, Ahn D, Johnson G, et al. Diffusion-tensor MR imaging of intracranial neoplasia and associated peritumoral edema: introduction of the tumor infiltration index. Radiology 2004;232:221-28

6. Morita K, Matsuzawa H, Fujii Y, et al. Diffusion tensor analysis of peritumoral edema using lambda chart analysis indicative of the heterogeneity of the microstructure within edema. J Neurosurg 2005;102:336-41

7. Yamasaki F, Kurisu K, Satoh K, et al. Apparent diffusion coefficient of human brain tumors at MR imaging. Radiology 2005;235:985-91

8. Calli $\mathrm{C}$, Kitis $\mathrm{O}$, Yunten N, et al. Perfusion and diffusion MR imaging in enhancing malignant cerebral tumors. Eur J Radiol 2006;58: $394-403$

9. Oh J, Cha S, Aiken AH, et al. Quantitative apparent diffusion coefficients and $\mathrm{T} 2$ relaxation times in characterizing contrast enhancing brain tumors and regions of peritumoral edema. J Magn Reson Imaging 2005;21:701-08

10. Wang W, Steward CE, Desmond PM. Diffusion tensor imaging in glioblastoma multiforme and brain metastases: the role of $p, q, L$, and fractional anisotropy. AJNR Am J Neuroradiol 2009;30:203-08

11. Reiche W, Schuchardt V, Hagen T, et al. Differential diagnosis of intracranial ring enhancing cystic mass lesions-role of diffusionweighted imaging (DWI) and diffusion-tensor imaging (DTI). Clin Neurol Neurosurg 2010;112:218-25

12. Wang S, Kim S, Chawla S, et al. Differentiation between glioblastomas and solitary brain metastases using diffusion tensor imaging. Neuroimage 2009;44:653-60

13. Chen XZ, Yin XM, Ai L, et al. Differentiation between brain glioblastoma multiforme and solitary metastasis: qualitative and quantitative analysis based on routine MR imaging. AJNR Am J Neuroradiol 2012;33:1907-12

14. Kinoshita M, Hashimoto N, Goto T, et al. Fractional anisotropy and tumor cell density of the tumor core show positive correlation in diffusion tensor magnetic resonance imaging of malignant brain tumors. Neuroimage 2008;43:29-35

15. Murakami R, Hirai T, Sugahara T, et al. Grading astrocytic tumors by using apparent diffusion coefficient parameters: superiority of a one- versus two-parameter pilot method. Radiology 2009;251: $838-45$

16. Stadlbauer A, Ganslandt O, Buslei R, et al. Gliomas: histopathologic evaluation of changes in directionality and magnitude of water diffusion at diffusion-tensor MR imaging. Radiology 2006;240:803-10

17. Al-Okaili RN, Krejza J, Woo JH, et al. Intraaxial brain masses: MR imaging-based diagnostic strategy-initial experience. Radiology 2007;243:539-50

18. Kim S, Pickup S, Hsu O, et al. Diffusion tensor MRI in rat models of invasive and well-demarcated brain tumors. NMR Biomed 2008;21: $208-16$

19. Beppu T, Inoue T, Shibata $Y$, et al. Fractional anisotropy value by diffusion tensor magnetic resonance imaging as a predictor of cell density and proliferation activity of glioblastomas. Surg Neurol 2005;63:56-61

20. Hope T, Westlye LT, Bjornerud A. The effect of gradient sampling schemes on diffusion metrics derived from probabilistic analysis and tract-based spatial statistics. Magn Reson Imaging 2012;30: 402-12

21. Jones DK. The effect of gradient sampling schemes on measures derived from diffusion tensor MRI: a Monte Carlo study. Magn Reson Med 2004;51:807-15

22. Landman BA, Farrell JA, Jones CK, et al. Effects of diffusion weighting schemes on the reproducibility of DTI-derived fractional anisotropy, mean diffusivity, and principal eigenvector measurements at 1.5T. Neuroimage 2007;36:1123-38

23. Wang S, Kim S, Chawla S, et al. Differentiation between glioblastomas, solitary brain metastases, and primary cerebral lymphomas using diffusion tensor and dynamic susceptibility contrast-enhanced MR imaging. AJNR Am J Neuroradiol 2011;32:507-14

24. Westin CF, Maier SE, Mamata H, et al. Processing and visualization for diffusion tensor MRI. Med Image Anal 2002;6:93-108

25. Wang S, Kim S, Zhang Y, et al. Determination of grade and subtype of meningiomas by using histogram analysis of diffusion-tensor imaging metrics. Radiology 2012;262:584-92

26. Rutter CM. Bootstrap estimation of diagnostic accuracy with patient-clustered data. Acad Radiol 2000;7:413-19

27. DeLong ER, DeLong DM, Clarke-Pearson DL. Comparing the areas under two or more correlated receiver operating characteristic curves: a nonparametric approach. Biometrics 1988;44:837-45

28. Toh CH, Wei KC, Ng SH, et al. Differentiation of tumefactive demyelinating lesions from high-grade gliomas with the use of diffusion tensor imaging. AJNR Am J Neuroradiol 2012;33:846-51

29. Toh $\mathrm{CH}, \mathrm{Wei} \mathrm{KC}, \mathrm{Ng} \mathrm{SH}$, et al. Differentiation of brain abscesses from necrotic glioblastomas and cystic metastatic brain tumors with diffusion tensor imaging. AJNR Am J Neuroradiol 2011;32: 1646-51

30. Zamecnik J. The extracellular space and matrix of gliomas. Acta Neuropathol 2005;110:435-42

31. Vargova L, Homola A, Zamecnik J, et al. Diffusion parameters of the extracellular space in human gliomas. Glia 2003;42:77-88

32. Pope WB, Mirsadraei L, Lai A, et al. Differential gene expression in glioblastoma defined by ADC histogram analysis: relationship to extracellular matrix molecules and survival. AJNR Am J Neuroradiol 2012;33:1059-64

33. Tropine A, Dellani PD, Glaser M, et al. Differentiation of fibroblastic meningiomas from other benign subtypes using diffusion tensor imaging. J Magn Reson Imaging 2007;25:703-08

34. Stylianopoulos T, Diop-Frimpong B, Munn LL, et al. Diffusion anisotropy in collagen gels and tumors: the effect of fiber network orientation. Biophys J 2010;99:3119-28

35. Preusser M, Capper D, Ilhan-Mutlu A, et al. Brain metastases: pathobiology and emerging targeted therapies. Acta Neuropathol 2012; 123:205-22

36. Varga I, Hutoczki G, Petras M, et al. Expression of invasion-related extracellular matrix molecules in human glioblastoma versus intracerebral lung adenocarcinoma metastasis. Cent Eur Neurosurg 2010;71:173-80 